\title{
The complete chloroplast genome sequence of Gentiana lawrencei var. farreri (Gentianaceae) and comparative analysis with its congeneric species
}

\author{
Peng-Cheng Fu ${ }^{1}$, Yan-Zhao Zhang ${ }^{1}$, Hui-Min Geng ${ }^{1}$, Shi-Long Chen ${ }^{\text {Corresp. } 2}$ \\ ${ }^{1}$ College of Life Science, Luoyang Normal University, Luoyang, China \\ ${ }^{2}$ Key Laboratory of Adaptation and Evolution of Plateau Biota, Northwest Institute of Plateau Biology, Chinese Academy of Sciences, Xining, China \\ Corresponding Author: Shi-Long Chen \\ Email address: slchen@nwipb.cas.cn
}

Background. The chloroplast (cp) genome is useful in plant systematics, genetic diversity analysis, molecular identification and divergence dating. The genus Gentiana contains 362 species, but there are only two valuable complete cp genomes. The purpose of this study is to report the characterization of complete cp genome of $G$. lawrencei var. farreri, which is endemic to the Qinghai-Tibetan Plateau (QTP).

Methods. Using high throughput sequencing technology, we got the complete nucleotide sequence of the $G$. lawrencei var. farreri cp genome. The comparison analysis including genome difference and gene divergence was performed with its congeneric species $G$. straminea. The simple sequence repeats (SSRs) and phylogenetics were studied as well.

Results. The $\mathrm{cp}$ genome of $\mathrm{G}$. lawrencei var. farreri is a circular molecule of $138,750 \mathrm{bp}$, containing a pair of 24,653 bp inverted repeats which are separated by small and large single-copy regions of 11,365 and 78,082 bp, respectively. The cp genome contains 130 known genes, including 85 protein coding genes (PCGs), eight ribosomal RNA genes and 37 tRNA genes. Comparative analyses indicated that $G$. lawrencei var. farreri is 10,241 bp shorter than its congeneric species G. straminea. Four large gaps were detected that are responsible for $85 \%$ of the total sequence loss. Further detailed analyses revealed that 10 PCGs were included in the four gaps that encode nine NADH dehydrogenase subunits. The cp gene content, order and orientation are similar to those of its congeneric species, but with some variation among the PCGs. Three genes, $n d h B, n d h F$ and $c l p P$, have high nonsynonymous to synonymous values. There are 34 SSRs in the $G$. lawrencei var. farreri cp genome, of which 25 are mononucleotide repeats: no dinucleotide repeats were detected. Comparison with the $G$. straminea cp genome indicated that five SSRs have length polymorphisms and 23 SSRs are species-specific. The phylogenetic analysis of 48 PCGs from 12 Gentianales taxa cp genomes clearly identified three clades, which indicated the potential of $\mathrm{cp}$ genomes in phylogenetics.

Discussion. The "missing" sequence of G. lawrencei var. farreri mainly consistent of ndh genes which could be dispensable under chilling-stressed conditions in the QTP. The complete cp genome sequence of G. lawrencei var. farreri provides intragenic information that will contribute to genetic and phylogenetic research in the Gentianaceae. 
1 The complete chloroplast genome sequence of Gentiana lawrencei var. farreri (Gentianaceae) and comparative analysis with its congeneric species

Peng-Cheng Fuํㄹ ${ }^{1}$ Yan-Zhao Zhang ${ }^{1}$, Hui-Min Geng ${ }^{1}$, Shi-Long Chen ${ }^{2}$

5

$6{ }^{1}$ College of Life Science, Luoyang Normal University, Luoyang, China

$7 \quad{ }^{2}$ Key Laboratory of Adaptation and Evolution of Plateau Biota, Northwest Institute of Plateau

8 Biology, Chinese Academy of Sciences, Xining, China

10 Corresponding Author:

11 Shi-Long Chen

1223 Xinning Road, Xining, Qinghai, 810008, China

13 Email address: slchen@nwipb.cas.cn 
14

15

\section{Introduction}

The chloroplast (cp) is the photosynthetic organelle that provides essential energy for plants, and is hypothesized to have arisen from ancient endosymbiotic cyanobacteria (Neuhaus \& Emes, 2000). In angiosperms, most cp genomes are circular DNA molecules, containing one large single-copy region (LSC), one small single-copy region (SSC) and a pair of inverted repeats (IRs) (Palmer, 1985; Jansen et al., 2005). The sizes of $\mathrm{cp}$ genomes in most angiosperms range from $120 \mathrm{~kb}$ to $160 \mathrm{~kb}$ caused by expansion of the IR regions and evolutionary contractions (Palmer, 1985; Wang et al., 2008).

Recently, the number of completely sequenced cp genomes from higher plants has increased significantly. The cp genome is useful in plant systematics research because of its maternal inheritance, haploid nature and highly conserved structures. It is widely used in the study of genetic diversity, molecular identification, phylogenetic classification and divergence dating (Shaw et al., 2007; Nikiforova et al., 2013; Carbonell-Caballero et al., 2015; Williams et al., 2016). The comparative analysis of $\mathrm{cp}$ genomes reveal insights into the $\mathrm{cp}$ genome evolution such as sequence inversion (Cho et al., 2015), gene loss (Wakasugi et al., 1994; Millen et al., 2001) and variation in borders of LSC, SSC and IR regions (Ni et al., 2016).

The family Gentianaceae has approximately 700 species (He, 1988) and is the third largest family of the Gentianales order in the Asterids clade. However, only one complete chloroplast genomes has been reported in this family so far (Ni et al., 2016). Gentiana is the largest genus in the Gentianaceae, containing 15 sections and about 362 species (Ho \& Liu, 2001). Gentiana plants have been widely used as traditional Chinese and Tibetan medicines (Ho \& Liu 2001) and 
are edificators in the Qinghai-Tibetan Plateau (QTP) alpine meadow. Although some studies have been carried out on the phylogenetics of Gentiana, they have all been based on one or several gene fragments (Yuan \& Küpfer, 1997; Yuan, Küpfer \& Doyle, 1996; Zhang et al, 2009). Together with their complicated evolutionary history (Yuan \& Küpfer, 1997), the phylogenetic relationships of Gentiana, especially intrasectional classification, remain controversial (Ho \& Liu, 2001; Favre et al., 2010). At present, there are only two complete $\mathrm{cp}$ genomes have been sequenced in the Gentiana: G. straminea and G. crassicaulis, which both belong to the same section, Cruciata Gaudin, and only G. straminea was reported (Ni et al., 2016). Therefore, it is necessary to develop genomic resources for Gentiana to provide valuable information to study their phylogenetic relationships and the evolutionary history of the genus.

Gentiana lawrencei var. farreri T. N. Ho is endemic to the QTP and belongs to sect. Kudoa (Masamune) Satake \& Toyokuni ex Toyokuni. It has very beautiful flowers and has been used in traditional Chinese and Tibetan medicine (Yang et al., 2012). Here, we report the cp genome sequence of G. lawrencei var. farreri and present a comparative analysis with its congeneric species G. straminea. The genome structure, insertions and deletions, repeat sequences and phylogenetics of Gentianaceae were analyzed. This study provided large amounts of sequence information for phylogenetic and evolutionary studies of Gentiana and the Gentianaceae.

\section{Materials and methods}

\section{Sample collection, genome sequencing, and assembly}

Gentiana lawrencei var. farreri was sampled in Qilian Mountain $\left(101^{\circ} 22^{\prime} 33^{\prime \prime} \mathrm{E}, 37^{\circ} 29^{\prime} 53^{\prime \prime} \mathrm{N}\right.$, Qinghai, China) from a single plant. Total genomic DNA was isolated from young leaves using a 
56

57

58

Dzup plant genomic DNA extraction kit (Sangon, Shanghai, China) following the manufacturer's instructions. After DNA isolation, the procedure was performed in accordance with the standard Illumina protocol, including sample preparation and sequencing. Approximately 5-10 $\mu \mathrm{g}$ of genomic DNA was fragmented using ultrasound, which was purified using the CASpure PCR Purification Kit (ChaoShi-Bio, Shanghai, China), followed end repair with poly-A on the $3^{\prime}$ ends. The DNA were then linked to adapters, extracted at specific size after agarose gel electrophoresis and amplified by PCR to yield a sequencing library. Then, a quarter of one flow-cell lane containing the fragmented genomic DNA of G. lawrencei var. farreri was sequenced using the Illumina HiSeq 4000 platform (Biomarker, Beijing, China), yielding 36.08 million 150-bp paired-end reads from a library of approximately 350-bp DNA fragments. Reads corresponding to plastid DNA were identified using a BLASTN (E-value: $10^{-6}$ ) search against the plastome sequences of two Gentiana taxa: G. straminea (GenBank accession no. NC_027441) and G. crassicaulis (NC_027442). A total of 2,517,802 reads (6.97\%) were recovered and assembled using Velvet 1.2.10 (Zerbino \& Birney, 2008). Eight contigs, ranging in size from 926 to $47,806 \mathrm{bp}$, were obtained. All the genomic regions located at the junction between the two contigs were verified by Sanger sequencing. The primers used were designed using PRIMER V5.0 and are provided in supplementary Table S1. The G. lawrencei var. farreri plastome sequence was deposited in GenBank (accession no. KX096882).

\section{Genome annotation}

The protein coding genes (PCGs), tRNAs and rRNAs in the cp genome were predicted and annotated using Dual Organellar GenoMe Annotator (DOGMA) using default parameters 
77 (Wyman, Jansen \& Boore, 2004). The positions of questionable start and stop codons, or intron

junctions of the PCGs, were verified using BLAST search against cp genomes of other closely related species. The cp gene map was drawn using OGDraw v1.2 (Lohse, Drechsel \& Bock, 2007). Simple sequence repeats (SSRs) were detected using MSDB 2.4 (http://msdb.biosv.com) with minimal repeat numbers of $10,5,4,3,3$, and 3 for mono-, di-, tri-, tetra-, penta-, and hexanucleotides, respectively.

\section{Comparative analysis with $G$. straminea}

The cp genome sequence from G. straminea (NC_027441) was obtained from the National Center for Biotechnology Information (NCBI). Genome comparison to identify the differences between G. lawrencei var. farreri and G. straminea was performed using mVISTA (Frazer et al., 2004) and Geneious Basic 5.6.4 (Kearse et al., 2012). Nonsynonymous (Ka) to synonymous (Ks) $(\mathrm{Ka} / \mathrm{Ks})$ ratios were calculated using DnaSP v5.10 (Librado \& Rozas, 2009).

\section{Phylogenetic analysis}

To illustrate the phylogenetic relationships of Gentiana with other major Gentianales clades with our cp genome sequence, the other 12 available complete $\mathrm{cp}$ genomes in the order were downloaded from GenBank (Table S2). Lactuca sativa from Asteraceae was used as outgroup. Forty-eight PCGs (atpA, atpB, atpE, atpH, atpI, cemA, matK, ndhD, ndhE, petA, petB, petD, pet $G, \operatorname{pet} L, p e t N, p s a A, p s a B, p s a I, p s a J, p s b A, p s b C, p s b D, p s b E, p s b F, p s b H, p s b I, p s b J, p s b K$, psbL, psbM, psbN, psbT, rbcL, rpl14, rpl16, rpl20, rpl22, rpl33, rpl36, rpoA, rps2, rps3, rps4, rps8, rps11, rps 14, rps 15 and rps18) found in all of the species were extracted from the selected cp genomes. The amino acid sequences of each of the $48 \mathrm{cp}$ PCGs were aligned using MSWAT 
98 (http://mswat.ccbb.utexas.edu/) with default settings, and back translated to nucleotide sequences.

99 Phylogenetic analyses were performed using the concatenated nucleotide sequences and

PhyML3.1 software (Guindon \& Gascuel, 2003) using the maximum likelihood (ML) method.

PhyML searches relied on the subtree pruning and regrafting (SPR) method with the GTR $+\mathrm{I}+\mathrm{G}$

model $(\mathrm{p}$-inv $=0.404$, gamma shape $=0.808)$, as determined using the Akaike information

criterion implemented in jModelTest 2.1 .7 (Guindon \& Gascuel, 2003; Posada, 2008). A

bootstrap analysis was performed with 100 replications.

105

106

107

108

109

110

111

112

113

114

115

116

117

118

\section{Results}

\section{The overall structure and general features of the $G$. lawrencei var. farreri cp genome}

The cp genome of G. lawrencei var. farreri is a closed circular molecule of 138,750 bp (Fig. 1), comprising a pair of IR regions (IRa and IRb) of 24,653 bp, one LSC region of 78,082 bp and one SSC region of 11,365 bp. It has an overall typical quadripartite structure that resembles the majority of land plant cp genomes (Shinozaki et al., 1986). The GC contents of the LSC, SSC, and IR regions and the whole cp genome are 35.7, 30.0, 43.6 and 38.0\%, respectively, which are similar to the other reported Gentiana cp genomes (Ni et al., 2016). The cp genome of $G$. lawrencei var. farreri contains 130 genes, including 85 PCGs accounting for 66,215 bp, and 37 tRNA and eight rRNA genes accounting for $11,781 \mathrm{bp}$. Among the 130 genes, 18 are located in the IR region. Most genes are present as a single copy, while all the rRNA genes and some of the tRNA and PCGs in the IR occur as double copies. A total of 84 unigenes were detected in the $\mathrm{cp}$ genome and this category is detailed in Table S3. Four genes each have one intron (atpF, rpoC1, $n d h B$ and $r p l 2)$ and two PCGs $(\operatorname{clp} P$ and $n d h F)$ and 1 ycf (ycf3) have two introns. Like most 
119

120

121

122

123

124

125

126

127

128

129

130

131

132

133

134

135

136

137

138

139

other land plants, rps 12 is trans-spliced, with its two $3^{\prime}$ end residues separated by an intron in the IR region, and the $5^{\prime}$ end exon is in the LSC region (Fig. 1). The 37 tRNAs contained 30 different tRNA genes and the eight rRNA genes contained four different tRNA genes. Both the number and types of the tRNAs are consistent with those presented in other species of vascular plants (Shinozaki et al., 1986).

\section{Comparison of $G$. lawrencei var. farreri and $G$. straminea cp genomes}

A comparative analysis between the $\mathrm{cp}$ genomes in Gentiana revealed that G. lawrencei var. farreri is $10,241 \mathrm{bp}$ shorter than that of $G$. straminea. As for the four parts of the cp genome, the LSC, SSC and IR of G. lawrencei var. farreri are 3185 bp, 5720 bp and 680 bp shorter than those of G. straminea, respectively (Table 1). Four big gaps (GapA-D) were detected: GapA (2241 bp) in the LSC, GapB (958 bp) in IRb, GapC (4582 bp) in the SSC and GapD (958 bp) in IRa. The four gaps represent $85 \%$ of the "missing" genome. All the gaps were verified by Sanger sequencing with primers designed using PRIMER V5 (Table S1). Compared with G. straminea, GapA contains three PCGs ( $n d h J, n d h K$ and $n d h C)$, GapB and GapD contain exon 2 of $n d h B$ and GapC contains five PCGs ( $n d h G, n d h I, n d h A$ and parts of $n d h E$ and $n d h H)$. A comparative analysis between $G$. lawrencei var. farreri and $G$. straminea cp genomes revealed that the sequence similarities between the $\operatorname{trn} H-G U G-p s b A, \operatorname{trnK}-U U U-\operatorname{trn} Q-U U G, \operatorname{trn} S-G C U-\operatorname{trn} G-$ GCC, atpH-atpI, rpoB-trnC-GCA, psbC-trnS-UGA, trnT-UGU-trnL-UAA, atpB-rbcL, ycfl-ndhF, rpl32-trnL-UAG and trnL-CAA-ycf15 intergenic regions are very low.

\section{Divergence hotspot}

The complete cp genomes of G. lawrencei var. farreri and G. straminea were compared using 
140 the mVISTA program to determine the level of sequence divergence. The comparison showed

141 that the coding regions of both $\mathrm{cp}$ genomes are highly conserved compared with the noncoding

142 regions. In particular, the intergenic regions showed the greatest divergence between the two cp

143 genomes. More divergence was found in the sequences of $c l p P, n d h B, n d h D, n d h E, n d h F$ and

$144 n d h H$, which are distributed mainly in the SSC regions, compared with other PCGs. The

145 nucleotide and amino acid sequences of the PCGs of $G$. lawrencei var. farreri and G. straminea

146 are highly similar, with average sequence similarities of 95.0 and $93.0 \%$, respectively. Between

147 the two species, the nucleotide sequence identities of the LSC, SSC, and IR are 88.7, 61.0, and

$14892.9 \%$, respectively. The most conserved genes include all the rRNA genes, the genes from

149 photosystem I, the cytochrome b/f complex genes and the ATP synthesis genes (Table S3 and 150 S4).

151 Divergence of coding gene sequence

152 Seventy-four PCGs are shared between the two species. Compared with G. straminea, 14 out of 153 the 74 shared PCGs had deletions and six had insertions (Table S4). The average Ks values 154 between the two Gentiana species were $0.0551,0.1133$, and 0.0243 in the LSC, SSC, and IR 155 regions, respectively, with a total average Ks of 0.0642 across all regions (Table S4). Although the coding region is highly conserved, we did observe slight variations. Based on the comparison of $\mathrm{Ka} / \mathrm{Ks}$ values among the regions, higher Ks values were observed for some genes, including rps8, rpl14, rpl36, rpl32, $n d h D, r p l 36$ and $n d h H$. The distribution of Ks values indicated that on average more of genes in the SSC region have experienced higher selection pressures than the rest regions of the cp genome. The $\mathrm{Ka} / \mathrm{Ks}$ ratio was also calculated, which was $>1$ for $n d h B$ in 
161 the IR region, $n d h F$ in SSC region and $c l p P$ from the LSC region (Fig. 2).

\section{SSR analysis}

163 Thirty-four SSR loci, 394 bp in length, were detected in the G. lawrencei var. farreri cp genome, 164 and there were 25, three, five, and two mono-, tri-, tetra-, and penta-nucleotide repeats, respectively (Table S5). No dinucleotide repeats were detected in the $\mathrm{cp}$ genome. Most of the SSRs are mononucleotide repeats, which is consistent with the study of George et al. (2015). Thirty of the 34 SSRs comprised A and T nucleotides, with a higher AT content $(95.9 \%)$ in these sequences compared with the rest of the genome. Among the SSRs, 23 were located in intergenic regions and 11 were found in coding genes, including those in the $\operatorname{css} A, \operatorname{rpoCl}, n d h F$, atpF, rpl32, matK, rpoA, atpB and psaB genes. Compared with G. straminea, six loci were identical, five were polymorphic, 28 were lost and 23 were specific to G. lawrencei var.farreri (Table S5).

\section{Phylogenetic relationship}

An ML phylogenetic tree constructed using 48 PCGs from 12 Gentianales taxa clearly identified

the three families (Gentianaceae, Rubiaceae and Apocynaceae) in the analysis as being monophyletic with high bootstrap value. (Fig. 3). The tree revealed that G. crassicaulis and G. straminea are more closely related to one another than either is to G. lawrencei var. farreri. All the nodes in the tree have high ( $>95 \%)$ bootstrap support.

Discussion

Evolution of G. lawrencei var. farreri

Much of the variation in the sequence complexity of angiosperm cp genomes appears to be the result of rather small length mutations. However, our comparative analysis showed that $G$. 
182 183

lawrencei var. farreri is 10,241 bp shorter than G. straminea. Although the cp genome size is variable, ranging from $120 \mathrm{~kb}$ to $160 \mathrm{~kb}$, huge genome losses in congeneric taxa are rarely reported. In general, most of the size changes in angiosperm cp genomes can be accounted for by rare deletions and duplications leading to massive changes in the size of the IR region (Palmer, 1985). This is not the case for G. lawrencei var. farreri and G. straminea. The total length variation mainly occurred in the SSC (5720 bp, 55.85\%) and LSC (3158 bp, 30.84\%) regions rather than the two IR regions (1360 bp, 13.28\%). More than half (50.33\%) of the sequence length in the SSC region was lost. Therefore, the cp genome size variation in the two Gentiana taxa was not caused by deletions in the IR regions, but by deletions in the SSC and LSC regions. Although the IR region can vary from 10 to $76 \mathrm{~kb}$ among angiosperms, in the great majority of species it is a rather constant $22-26 \mathrm{~kb}$ in size (Palmer, 1985). The junction between the IR and LSC region is located within the rps19 gene in G. lawrencei var. farreri, similar to majority of dicots and some monocots (Wang et al., 2008; Ni et al., 2016). The more or less fixed position of IR-LSC junction within a coding gene suggests some selection is operating to constrain the boundaries of the IR (Palmer, 1985). It contributes to the more constant size of the IRs than the LSC and SSC region in the great majority of angiosperms.

The SSC region of G. lawrencei var. farreri has experienced drastic variation as compared to its congeneric species. Compared with G. straminea, the SSC region contributes $55.85 \%$ of the cp genome sequence length variation and only showed $61.0 \%$ nucleotide identity. The SSC region also has a much higher Ks (0.1133) value than the LSC (0.0551) and IR (0.0243) regions. Two possible explanations about variation in the SSC region were proposed in previous studies. 
203 Firstly, the higher rate of molecular evolution in the SSC than other regions was also observed in

204 Walker, Zanis \& Emery (2014) who attributed it to low proportion of coding vs. noncoding 205 regions in the sequence. However, this does not appear to be true in our study. Secondly, the 206 SSC region is a "hotspot" for recombination (Palmer, 1983; Liu et al., 2013; Walker et al., 2015).

207 We did not yet detect inversion in the SSC region of G. lawrencei var. farreri. Therefore, the 208 drastic variation may be result of other reasons. The functional genes associated with the variation in the SSC region of $G$. lawrencei var. farreri, mainly focus on the $n d h$ genes, might provide an insight into the reasons for the drastic variation.

211 In chloroplasts, gene loss is an ongoing process (Martin et al., 1998). The huge genome loss in G. lawrencei var. farreri was mainly accounted for by four big gaps, which caused the loss of the entire $n d h J, n d h K, n d h C, n d h E, n d h G, n d h I$, and $n d h A$ genes and partial loss of $n d h H$ and $n d h B$. The protein products of all the lost genes are NADH dehydrogenase (NDH) subunits. The $\mathrm{cp}$ DNA of most of the higher plants contains $11 n d h$ genes, which encode protein subunits of the thylakoid NDH complex. The complex is analogous to mitochondrial complex I (EC 1.6.5.3), which catalyzes the transfer of electrons from NADH to plastoquinone (Sazanov, Burrows \& Nixon, 1998). The cp $n d h$ genes have been retained in most higher plants (Martín \& Sabater, 2010), but appear to have been lost frequently in parasitic and epiphytic plants (e.g. Stefanovi \& Olmstead, 2005) along with other cp genes apparently associated with a loss of or reduction in photosynthetic capability (Iles, Smith \& Graham, 2013). Although the $n d h$ genes could be dispensable under mild non-stressing environments, transgenic plants defective in $n d h$ genes showed that the NDH complex is required to optimize photophosphorylation rates and showed 
224 impaired photosynthesis rates under stress conditions (Marín \& Sabater, 2010). Cyclic 225 photophosphorylation via the NDH pathway might play an important role in regulating $\mathrm{CO}_{2}$ 226 assimilation under heat-stress conditions, but is less important under chilling-stressed conditions 227 (Wang et al., 2006). Therefore, the absence of NDH in G. lawrencei var. farreri is understandable when considering the cool conditions in the QTP, which is the natural habitat of Gentiana (Ho \& Liu, 2001). Meanwhile, the $n d h$ loss between two congeneric species might offer a clue to the divergence and evolution of Gentiana.

Variation in the divergence of the coding region was observed between the two Gentiana species. Although the coding region was generally highly conserved, the $r p s 8, r p l 14$, and $r p l 36$ genes of the LSC region and the $r p l 32, n d h D, n d h F$, and $n d h H$ genes of the SSC region of $G$. lawrencei var. farreri showed a higher evolution rate compared with other genes. Based on the sequence identity among the three regions, the IR region is more conserved than the LSC and

SSC regions. This agrees with previous studies that hypothesized that the frequent recombinant events occurring in the IR region result in selective constraints on sequence homogeneity, causing them to diverge at a slower rate than the LSC and SSC regions (Qian et al., 2013; Cho et al., 2015). Our data confirm a positive selection pressure at the protein coding genes. The $n d h B$ gene of the IR region, $n d h F$ of the SSC region and $c l p P$ from the LSC region of G. lawrencei var. farreri presented higher $\mathrm{Ka} / \mathrm{Ks}$ ratios $(>1.0)$, indicating that they had evolved under positive selection. The clpP gene also showed a high $\mathrm{Ka} / \mathrm{Ks}$ ratio in Fagopyrum tataricum (Cho et al., 2015). Interestingly, the $n d h B$ and $n d h F$ genes experienced positive selection pressure. In the absence of nine $n d h$ genes in $G$. lawrencei var. farreri, the remaining $n d h B$ and $n d h F$ genes 
might play an important role in cyclic photophosphorylation, although the functions of $n d h B$ and

246

247 $n d h F$ genes are unknown. The $n d h B$ and $n d h F$ genes are probably transcribed independently as monocistronic mRNAs (Martín \& Sabater, 2010). Favory et al. (2005) proposed that the transcription of the $n d h F$ gene requires the nuclear-encoded sigma4 factor; the $n d h F$ product in turn would stimulate the transcription of the other plastid $n d h$ genes. Therefore, the selection pressure on the $n d h F$ gene may play an important role in evolution of $n d h$ genes.

\section{Phylogenetic value}

The ML phylogenetic tree of Gentianales constructed using 48 PCGs clearly grouped the taxa from the three families into three clades. The phylogenetic relationships were consistent with previous studies that classified the three families as three monophyletic clades and identified the Rubiaceae as the base group in the Gentianales (Backlund, Oxelman \& Bremer, 2000). The cp genome has also been used successfully for phylogenetic reconstruction in several studies (Carbonell-Caballero et al., 2015; Williams et al., 2016). In Gentiana, several phylogeny studies have been carried out (Yuan \& Küpfer, 1997; Mishiba et al., 2009; Zhang et al., 2009). However, these studies were all based on one or several DNA fragments, which, together with their complicated evolutionary history, have led to the phylogenetic relationships of Gentiana being controversial due to inconsonant sectional classification and the low support for relationships (Ho \& Liu 2001; Favre et al., 2010). For example, the sect. Chondrophyllae, which has 10 series and 163 species, derived within a very short period of time followed by subsequent rapid radiation (Yuan \& Küpfer, 1997), making the infrasectional phylogenetic relationships of this section difficult to determine. In addition, previous phylogenetic analyses based on internal 
266 transcribed spacer regions reclassified five clades in sect. Cruciata but failed to find 267 corresponding morphological circumscriptions to support them (Zhang et al., 2009). Our analysis 268 also identified substantial length variation and amount of base substitutions in the cp genome 269 between two species of Gentiana; therefore, to realize the full potential of the cp genome in phylogenetic analysis, more taxa of different secttions should be included in the cp genome comparison analysis.

Chloroplast SSRs are good tools for studies in plant ecology and evolution (Provan, Powell \& Hollingsworth, 2001). Microsatellites often show high levels of polymorphism and are thus used widely in studies of genetics and evolution. However, SSRs in the nuclear genome are usually species-specific and are thus used mainly for intraspecific genetic studies rather than phylogenetic studies of related species. Unlike nuclear SSRs, chloroplast SSRs are frequently cross-amplified in related species and thus could be used for phylogenetic studies (Provan,

Powell \& Hollingsworth, 2001). We detected five polymorphic SSRs between G. lawrencei var.

farreri and G. straminea, which belong to different sections. SSRs are more polymorphic than cp loci that are amplified by universal primers; therefore, the polymorphic SSRs could offer higher resolution for phylogenetic tree construction in Gentiana.

\section{Conclusion}

We present the first report of the complete cp genome sequence of G. lawrencei var. farreri and describe its evolutionary characteristics in comparison with G. straminea. About $10 \mathrm{~kb}$ sequence which mainly consistent of $9 n d h$ genes were lost in G. lawrencei var. farreri. The divergence 
287 hotspots and SSRs clarified here could be used as molecular markers and will be useful for 288 further studies on population genetics, phylogenetics and evolution of the genus Gentiana.

Acknowledgments

291

We thank Shan-shan Sun of the Wuhan Botanical Garden, Chinese Academy of Sciences, for providing laboratory support.

\section{References}

295

296

297

298

299

300

301

302

303

304

305

306

307

Backlund M, Oxelman B, Bremer B. 2000. Phylogenetic relationships within the Gentianales based on ndhF and rbcL sequences, with particular reference to the Loganiaceae. American Journal of Botany, 87(7): 1029-1043. DOI: 10.2307/2657003.

Bohnert HJ, Crouse EJ, Schmitt JM. 1982. Chloroplast genome organization and RNA synthesis. Encyclopedia Plant Physiol B, 14: 475-530.

Carbonell-Caballero J, Alonso R, Ibañez V, Terol J, Talon M, Dopazo J. 2015. A phylogenetic analysis of 34 chloroplast genomes elucidates the relationships between wild and domestic species within the genus Citrus. Molecular Biology and Evolution, 32(8): 2015-2035. DOI: 10.1093/molbev/msv082.

Cho KS, Yun BK, Yoon YH, Hong SY, Mekapogu M, Kim KH, Yang TJ. 2015. Complete chloroplast genome sequence of tartary buckwheat (Fagopyrum tataricum) and comparative analysis with common buckwheat (F. esculentum). PloS one, 10(5), e0125332. DOI: 10.1371/journal.pone.0125332. 
308 Favre A, Yuan YM, Küpfer P, Alvarez N. 2010. Phylogeny of subtribe Gentianinae 309 (Gentianaceae): biogeographic inferences despite limitations in temporal calibration points. Taxon, 59(6): 1701-1711. DOI: 10.2307/41059867.

311

312

313

314

315

316

317

318

319

320

321

322

323

324

325

326

327

328

Favory JJ, Kobayshi M, Tanaka K, Peltier G, Kreis M, Valay JG, Lerbs-Mache S. 2005. Specific function of a plastid sigma factor for ndhF gene transcription. Nucleic Acids Research, 33(18): 5991-5999. DOI: 10.1093/nar/gki908.

George B, Bhatt BS, Awasthi M, George B, Singh AK. 2015. Comparative analysis of microsatellites in chloroplast genomes of lower and higher plants. Current Genetics, 61(4), 665-677. DOI: 10.1007/s00294-015-0495-9.

Guindon S, Gascuel O. 2003. A simple, fast and accurate method to estimate large phylogenies by maximum-likelihood. Systematic Biology, 52: 696-704. DOI: 10.1080/10635150390235520.

He TN. 1988. Sect. Cruciata. In: He, T.N. (Ed.), Flora Reipublicae Popularis Sinicae 62. Gentianaceae. Science Press, Beijing, China, pp. 1-75.

Ho TN, Liu SW. 2001. A worldwide monograph of Gentiana. Beijing: Science Press.

Frazer KA, Pachter L, Poliakov A, Rubin EM, Dubchak I. 2004. VISTA: computational tools for comparative genomics. Nucleic acids research, 32(suppl 2): W273-W279. DOI: 10.1093/nar/gkh458.

Iles WJ, Smith SY, Graham SW. 2013. A well-supported phylogenetic framework for the monocot order Alismatales reveals multiple losses of the plastid NADH dehydrogenase complex and a strong long-branch effect. Early events in monocot evolution, 1-28. 
329

330

331

332

333

334

335

336

337

338

339

340

341

342

343

344

345

346

347

348

349

Jansen RK, Raubeson LA, Boore JL, dePamphilis CW, Chumley TW, Haberle RC, Wyman SK, Alverson AJ, Peery R, Herman SJ, Fourcade HM, Kuehl JV, McNeal JR, Leebens-Mack J, Cui L. 2005. Methods for obtaining and analyzing chloroplast genome sequences. Methods Enzymol, 395: 348-384. DOI: 10.1016/S0076-6879(05)95020-9.

Kearse M, Moir R, Wilson A, Stones-Havas S, Cheung M, Sturrock S, Buxton S, Cooper A, Markowitz S, Duran C, Thierer T, Ashton B, Meintjes P, Drummond A. 2012. Geneious Basic: an integrated and extendable desktop software platform for the organization and analysis of sequence data. Bioinformatics, 28(12): 1647-1649. DOI: 10.1093/bioinformatics/bts 199.

Kurtz S, Schleiermacher C. 1999. REPuter: fast computation of maximal repeats in complete genomes. Bioinformatics, 15 (5): 426-427. DOI: 10.1093/bioinformatics/15.5.426.

Librado P, Rozas J. 2009. DnaSP v5: a software for comprehensive analysis of DNA polymorphism data. Bioinformatics, 25(11): 1451-1452. DOI: 10.1093/bioinformatics/btp187.

Liu Y, Huo N, Dong L, Wang Y, Zhang S, Young HA, Feng X, Gu YQ. 2013. Complete chloroplast genome sequences of Mongolia medicine Artemisia frigida and phylogenetic relationships with other plants. PLoS One 8: e57533. DOI: 10.1371/journal.pone.0057533

Lohse M, Drechsel O, Bock R. 2007. OrganellarGenomeDRAW (OGDRAW): a tool for the easy generation of high-quality custom graphical maps of plastid and mitochondrial genomes. Current genetics, 52: 267-274. DOI: 10.1007/s00294-007-0161-y.

Martin W, Stoebe B, Goremykin V, Hansmann S, Hasegawa M, Kowallik KV. 1998. Gene 
Martín M, Sabater B. 2010. Plastid ndh genes in plant evolution. Plant Physiology and Biochemistry, 48(8): 636-645. DOI: 10.1016/j.plaphy.2010.04.009.

354

355

356

357

358

359

360

361

362

363

364

365

366

367

368

369

370

Millen RS, Olmstead RG, Adams KL, Palmer JD, Lao NT, Heggie L, Kavanagh TA, Hibberd JM, Gray JC, Morden CW, Calie PJ, Jermiin LS, Calie PJ. 2001. Many parallel losses of infA from chloroplast DNA during angiosperm evolution with multiple independent transfers to the nucleus. The Plant Cell, 13(3): 645-658.

Mishiba KI, Yamane K, Nakatsuka T, Nakano Y, Yamamura S, Abe J, Kawamura H, Takahata Y, Nishihara M. 2009. Genetic relationships in the genus Gentiana based on chloroplast DNA sequence data and nuclear DNA content. Breeding Science, 59(2): 119-127. DOI: 10.1270/jsbbs.59.119.

Neuhaus HE, Emes MJ. 2000. Nonphotosynthetic metabolism in plastids. Annual Review of Plant Biology, 51(1): 111-140. DOI: 10.1146/annurev.arplant.51.1.111.

Ni L, Zhao Z, Xu H, Chen S, Dorje G. 2016. The complete chloroplast genome of Gentiana straminea (Gentianaceae), an endemic species to the Sino-Himalayan subregion. Gene, 577(2): 281-288. DOI: 10.1016/j.gene.2015.12.005.

Nikiforova SV, Cavalieri D, Velasco R, Goremykin V. 2013. Phylogenetic analysis of 47 chloroplast genomes clarifies the contribution of wild species to the domesticated apple maternal line. Molecular Biology and Evolution, 30(8): 1751-1760. DOI: 10.1093/molbev/mst092. 
371 Palmer JD. 1983. Chloroplast DNA exists in two orientations. Nature, 301: 92-93. DOI:

372

373

374

375

376

377

378

379

380

381

382

383

384

385

386

387

388

389

390

391

10.1038/301092a0.

Palmer JD. 1985. Comparative organization of chloroplast genomes. Annual review of genetics, 19(1): 325-354. DOI: 10.1146/annurev.ge.19.120185.001545.

Posada D. 2008. jModelTest: phylogenetic model averaging. Molecular biology and evolution, 25(7): 1253-1256. DOI: 10.1093/molbev/msn083.

Provan J, Powell W, Hollingsworth PM. 2001. Chloroplast microsatellites: new tools for studies in plant ecology and evolution. Trends in Ecology \& Evolution, 16(3): 142-147. DOI: 10.1016/S0169-5347(00)02097-8.

Qian J, Song JY, Gao HH, Zhu YJ, Xu J, Pang XH, Yao H, Sun C, Li X, Li CY, Liu JY, Xu HB, Chen SL. 2013. The complete chloroplast genome sequence of the medicinal plant Salvia miltiorrhiza. PloS one, 8(2), e57607. DOI: 10.1371/journal.pone.0057607.

Sazanov LA, Burrows PA, Nixon PJ. 1998. The plastid ndh genes code for an NADH-specific dehydrogenase: isolation of a complex I analogue from pea thylakoid membranes. Proceedings of the National Academy of Sciences, 95(3): 1319-1324.

Shaw J, Lickey, EB, Schilling EE, Small RL. 2007. Comparison of whole chloroplast genome sequences to choose noncoding regions for phylogenetic studies in angiosperms: the tortoise and the hare III. American Journal of Botany, 94(3): 275-288. DOI: 10.3732/ajb.94.3.275.

Shinozaki K, Ohme M, Tanaka M, Wakasugi T, Hayashida, N, Matsubayashi T, Zaita N Chunwongse J, Obokata J, Yamaguchi-Shinozaki K, Ohto C, Torazawa K, Meng BY, Sugita M, Deno H, Kamogashira T, Yamada K, Kusuda J, Takaiwa F, Kato A, Tohdoh N, 
392

393

394

395

396

397

398

399

400

401

402

403

404

405

406

407

408

409

410

411

412

Shimada H, Ohto C. 1986. The complete nucleotide sequence of the tobacco chloroplast genome: its gene organization and expression. The EMBO journal, 5(9): 2043-2049. DOI: 10.1007/BF02669253.

Stefanovi S, Olmstead RG. 2005. Down the slippery slope: plastid genome evolution in convolvulaceae. Journal of Molecular Evolution, 61(3): 292-305. DOI: 10.1007/s00239004-0267-5.

Wakasugi T, Tsudzuki J, Ito S, Nakashima K, Tsudzuki T, Sugiura M. 1994. Loss of all $n d h$ genes as determined by sequencing the entire chloroplast genome of the black pine Pinus thunbergii. Proceedings of the National Academy of Sciences, 91(21): 9794-9798. DOI: 10.1073/pnas.91.21.9794.

Walker JF, Zanis MJ, Emery NC. 2014. Comparative analysis of complete chloroplast genome sequence and inversion variation in Lasthenia burkei (Madieae, Asteraceae). American Journal of Botany, 101(4): 722-729. DOI: 10.3732/ajb.1400049.

Walker JF, Jansen RK, Zanis MJ, Emery NC. 2015. Sources of inversion variation in the small single copy (SSC) region of chloroplast genomes. American Journal of Botany, 102 (11): 12. DOI:10.3732/ajb.1500299.

Wang P, Duan W, Takabayashi A, Endo T, Shikanai T, Ye JY, Mi H. 2006. Chloroplastic NAD (P) $\mathrm{H}$ dehydrogenase in tobacco leaves functions in alleviation of oxidative damage caused by temperature stress. Plant Physiology, 141(2): 465-474. DOI: 10.1104/pp.105.070490.

Wang RJ, Cheng CL, Chang CC, Wu CL. Su TM, Chaw SM. 2008. Dynamics and evolution of the inverted repeat-large single copy junctions in the chloroplast genomes of monocots. 
BMC Evolutionary Biology, 8(1): 36. DOI: 10.1186/1471-2148-8-36.

414 Williams AV, Miller JT, Small I, Nevill PG, Boykin, LM. 2016. Integration of complete 415 chloroplast genome sequences with small amplicon datasets improves phylogenetic 416 resolution in Acacia. Molecular phylogenetics and evolution, 96: 1-8. DOI: 10.1016/j.ympev.2015.11.021.

Wyman SK, Janse, RK, Boore JL. 2004. Automatic annotation of organellar genomes with DOGMA. Bioinformatics, 20(17): 3252-3255. DOI: 10.1093/bioinformatics/bth352.

Yang AM, Sun J, Han H, Shi XL, Xu GQ, Zhang XR. 2012. Chemical constituents from Gentiana farreri Balf. f. Chinese Traditional Patent Medicine, 4: 506-508.

Yuan YM., Kupfer P, Doyle JJ. 1996. Infrageneric phylogeny of the genus Gentiana (Gentianaceae) inferred from nucleotide sequences of the internal transcribed spacers (ITS) of nuclear ribosomal DNA. American Journal of Botany, 83: 641-652. DOI: $10.2307 / 2445924$.

Yuan YM, Küpfer P. 1997. The monophyly and rapid evolution of Gentiana sect. Chondrophyllae Bunge sl (Gentianaceae): evidence from the nucleotide sequences of the internal transcribed spacers of nuclear ribosomal DNA. Botanical Journal of the Linnean, 123: 25-43. DOI: 10.1111/j.1095-8339.1997.tb01403.x.

Zerbino DR, Birney E. 2008. Velvet: algorithms for de novo short read assembly using de Bruijn graphs. Genome research, 18(5): 821-829. DOI: 10.1101/gr.074492.107. 
datasets. Taxon, 58(3): 862-870.

435 


\section{Table $\mathbf{1}$ (on next page)}

Comparison of genome contents of $G$. lawrencei var. farreri and G. straminea. 


\begin{tabular}{lll}
\hline & G. lawrencei var. farreri & G. straminea \\
\hline Total Sequence Length (bp) & 138,750 & 148,991 \\
Large Single Copy (bp) & 78,082 & 81,240 \\
Inverted Repeat Region (bp) & 24,653 & 25,333 \\
Small Single Copy (bp) & 11,365 & 17,085 \\
GC Content (\%) & 38 & 37.7 \\
Total CDS Bases (bp) & 66,215 & 75,780 \\
Average CDS Length (bp) & 779 & 758 \\
Total RNA Bases (bp) & 11,781 & 11861 \\
Average Intergenic Distance (bp) & 467 & 403 \\
\hline
\end{tabular}

2 
Figure 1

Map ofthechloroplast genome of $G$. lawrencei var. farreri.

Genes drawn inside the circle are transcribed clockwise, and those outside are transcribed counterclockwise. Genes belonging to different functional groups are shown in different colors.

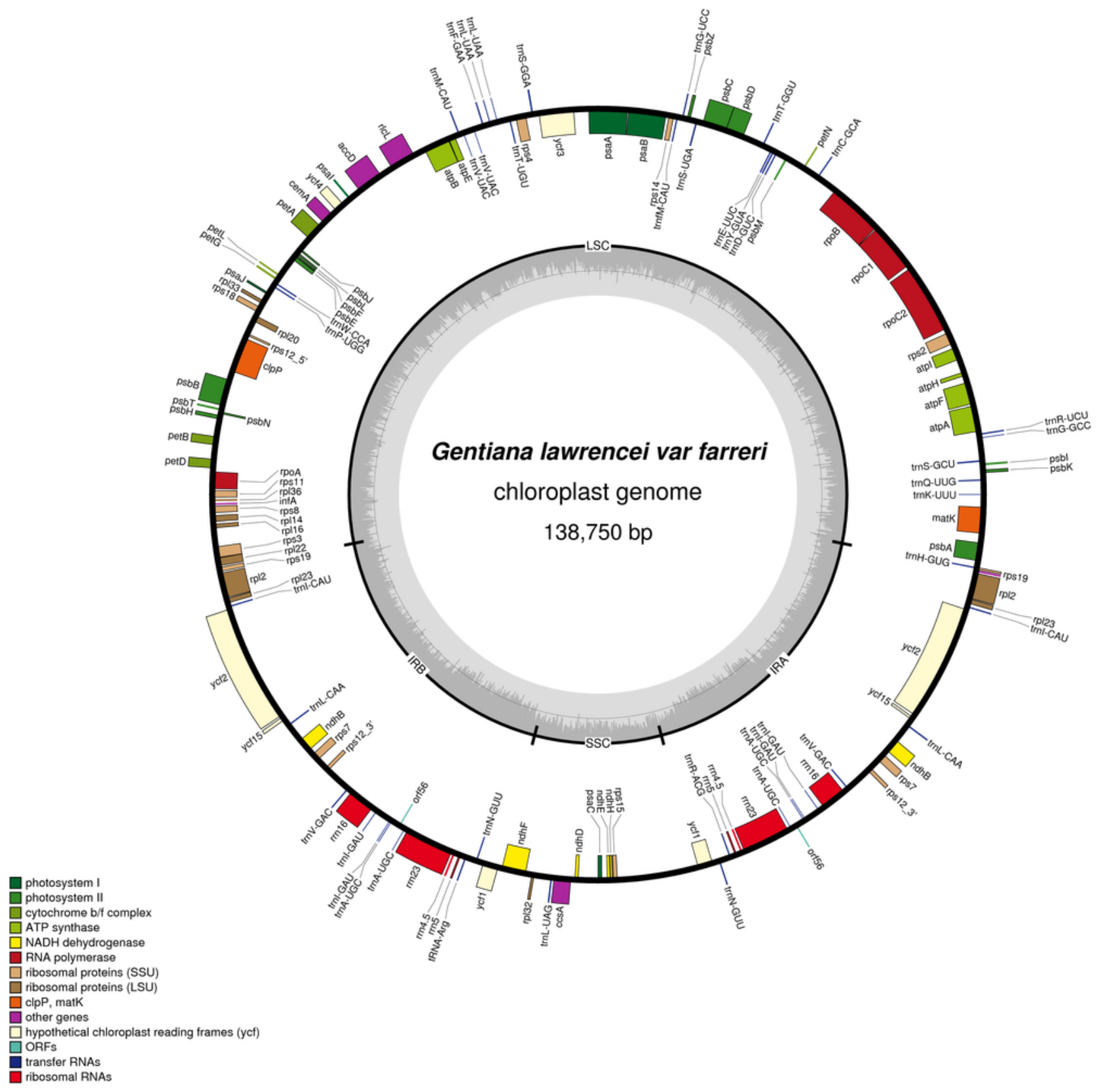




\section{Figure 2 (on next page)}

Gene-specific Ka/Ks ratios between the chloroplast genomes of two Gentiana species (G. lawrencei var. farreri and G. straminea).

Three genes (clpP, ndhB and $n d h F)$ returned $\mathrm{Ka} / \mathrm{Ks}$ ratios greater than 1.0, whereas the $\mathrm{Ka} / \mathrm{Ks}$ ratios of the other genes were less than 1.0. 


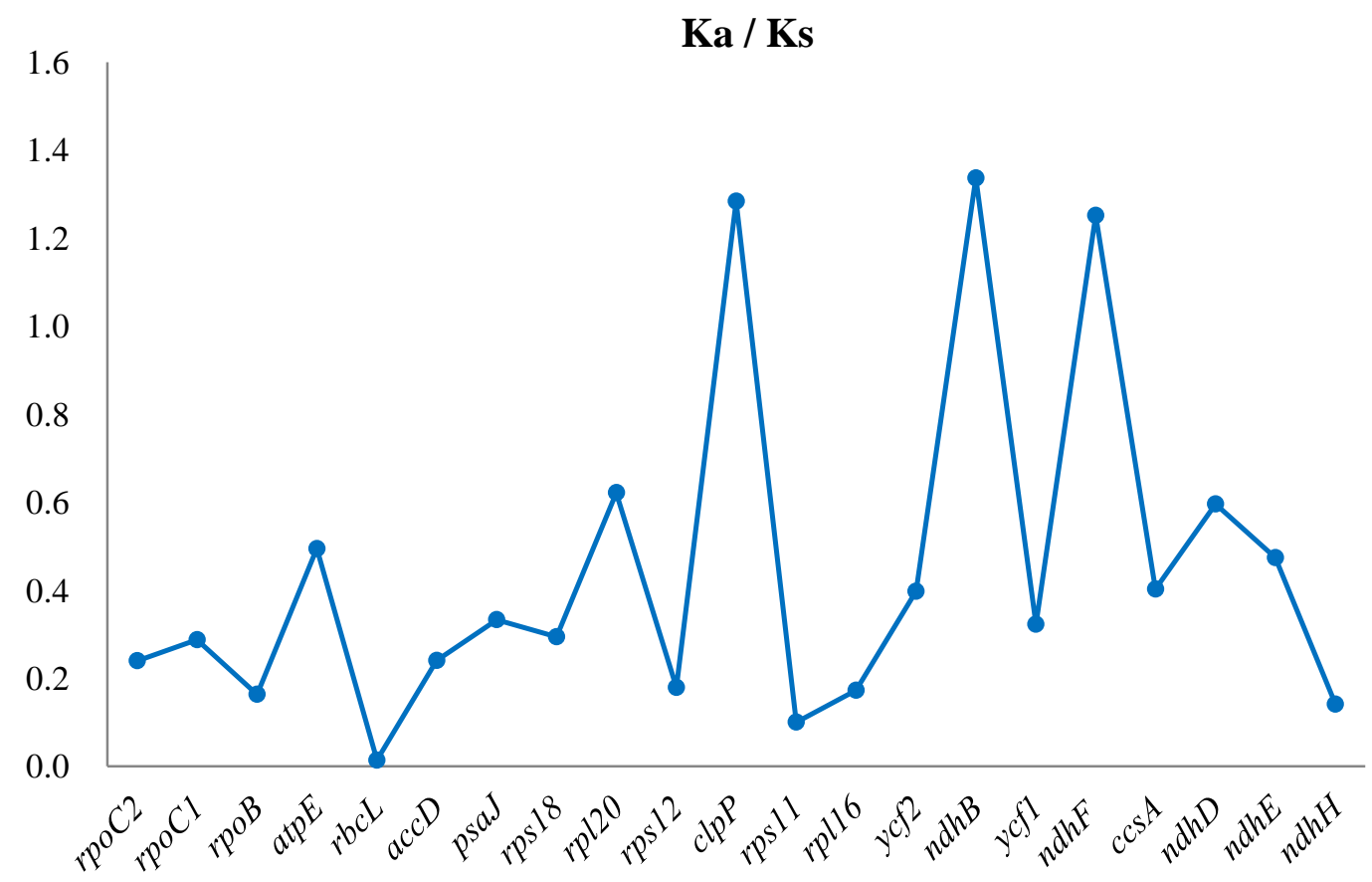


Figure 3

Phylogenetic analysis of 12 Gentianales species using 48 CDS regions of the chloroplast genomes.

Data sources: Gentiana straminea (NC_027441); Gentiana crassicaulis (NC_027442);

Catharanthus roseus (NC_021423); Rhazya stricta (NC_024292); Nerium oleander

(NC_025656); Pentalinon luteum (NC_025658); Oncinotis tenuiloba (NC_025657); Cynanchum auriculatum (NC_029460); Asclepias syriaca (NC_022432); Coffea arabica (NC_008535); Morinda officinalis (NC_028009) and Lactuca sativa (NC_007578).

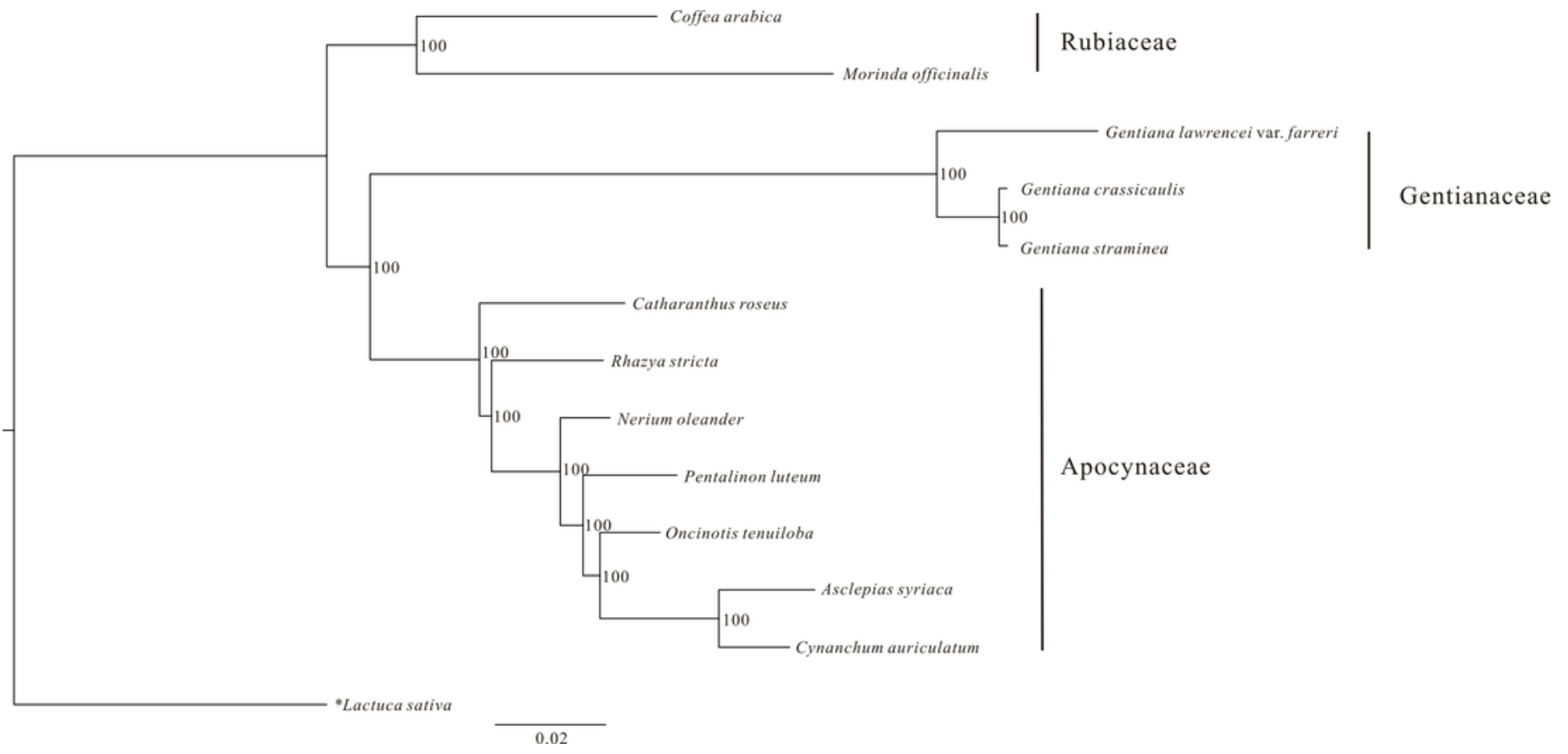

